\title{
The Mystery of Spherules on Stems
}

\author{
The formation process of the martian "blueberries" on stems
}

\author{
Charles W. Shults III \\ Shults Laboratories Inc.
}

\begin{abstract}
The Mars Exploration Rover Opportunity located many spherules that were on thin stalks or stems. The spherules appear to be formed inside sedimentary deposits and are thought by many to be hematite concretions or nodules. These spherules are expected to be inert but their appearance shows that some process is creating these stalks. It is our contention that the formation of the stalks has little to do with the spherules themselves, but instead is the result of an erosive process driven by the martian environment. We will demonstrate that the stalks have a simple origin that relies only on the intrinsic hardness of the spherules versus the less durable sedimentary substrate. These findings will indicate a set of conditions on Mars that must be present, and clear evidence will be shown to support this conclusion.
\end{abstract}

There has been a long-running mystery that was only discovered in early 2004 as the MER began exploring the surface of Mars. The findings of millions of spherules, considered to be hematite concretions, was one major result of the mission. While the composition of the spherules is still only approximately known, we have enough information to make some reasonable assumptions about them.

Not long after finding the spherules, it was found that in many places they appeared to be on stems or stalks. This quickly fueled speculation about a possible fungal explanation ${ }^{\mathrm{i}}$ and that perhaps the spherules had grown these extensions, but on closer examination, it became clear that this was not true. The spherules are not active in a biological sense, so another explanation had to be found.

Here we will present a simple theory that clearly explains the phenomenon and what the conditions for their formation must be.

\section{Chemistry of the spherules and sediments}

Analysis methods are limited for the MER but what is known is that the spherules appear to be roughly $35 \%$ to $45 \%$ hematite ${ }^{\mathrm{ii}}$. It has been proposed that they started out as carbonate nodules that were later replaced by mineral recrystallization ${ }^{\mathrm{iii}}$ (a water-driven replacement process) to become what we see today.

The sedimentary matrix that the spherules are seen to come from appears to be a composition of basalt-derived clay, fine-grained sandstone and sulfate minerals along with some small percentage of fine silica particles. It is theorized that this sediment formed at the bottom of a shallow sea or lake containing brine based on sulfate salts and some chlorides ${ }^{\text {iv }}$.

Readings from the rover instruments also identify the sedimentary rock as having the signature of basaltic rock, which might seem paradoxical. Basalt does not typically erode into sand-like particles easily, but often tends to become clay through a process of hydrolysis. When the composition is correct however, it will form rough, blocky particles of sand. This is dependent on the silica and alkali metal content of the basalt, among other things, but the elemental composition of weathered basalt appears to be similar to the components in the sediments in Meridianiv .

There is some evidence from the RAT (rock abrasion tool) and the rover wheels themselves that this sedimentary material is relatively soft. Its optical properties indicate that it is a bright white to pale orange on the interior while being colored orange or brown by the dust present in the atmosphere and 
the soil. The weight of the rover has been used to fracture the sediment and explore its interior to some extent.

It can be seen directly that the spherules are distributed widely over the surface of the soil in the region of Meridiani Planum, and often on the surface of the sedimentary slabs. If we examine the sediment itself we see a rather uniform distribution of spherules throughout the matrix rock, indicating that their deposition and/or formation was a constant, ongoing process.

As the sediment erodes, spherules are exposed and eventually released, resulting in the huge population of them that the images show us. So, the sediments appear to be the source of the spherules and the erosion of those sediments releases them. Now we can examine the phenomenon of the stems.

\section{What are the stems?}

The stems are apparent mineral formations that extend from the sedimentary matrix and are tipped by a spherule. Most of them are quite short but some are as much as five times the diameter of a spherule in length.

Images of the stems first became very clear around sol 85 as Opportunity passed by Fram Crater. In this area it was easy to locate hundreds of examples of this phenomenon with data across the spectrum and in stereo. The stems were from a few millimeters in length to as much as three times the diameter of a spherule. Most of the spherules are only a few millimeters in diameter, averaging about 3.5 millimeters, so even at the largest these stems were about 10 to 11 millimeters.

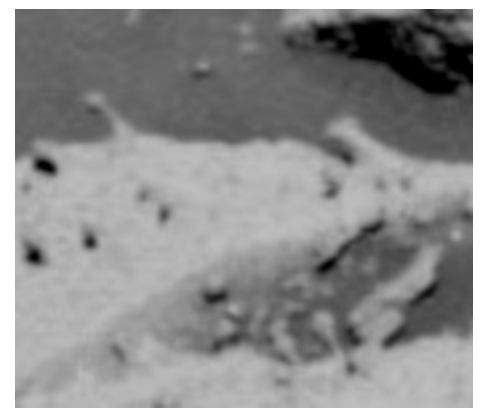

Figure $1 A$ and $1 B:$ The left image (1A) shows stems from sol 85 . The anaglyph image (1B) allows us to see the extension of a large stem without background clutter.

In later images from around sols 161 to 165, stems are seen that are much more extended, with lengths of about 15 to 18 millimeters. The physical structure of the stems presents an appearance identical to forms that would result from sandblasting a soft medium with a more durable obstruction in the abrasive flow path. This is a solid clue to the process.

The immediate theory would be that a flow of some carrier fluid would work against the sediment surface, producing the smooth, rounded appearance. Examination of the sites where stems are common shows evidence that a long-term directed flow of fluid has been in operation. There is a visible trail in the area of the effect which sometimes requires constructing an image of the area using numerous adjacent frames of image data.

Due to the fact that flat images cannot fully convey the depth and shape of this evidence, it is often necessary to use stereo images. Anaglyphs are being used for that purpose in this paper.

\section{Fluids that are reasonably expected to be present}

There are three candidate fluids that could produce these effects. Each has specific properties which can be helpful in finding the proper solution.

The first obvious possibility is the atmosphere. Air pressure on Mars is generally very low when compared to the terrestrial environment. Typical air pressures range from a minimum of roughly 
5 millibars to a maximum of about 10 millibars, dependent on surface elevation vi . At "sea level" on Mars, the pressure is very close to 6.5 millibars. Opportunity is at an elevation of -4.2 kilometers from zero elevation, yielding an average local atmospheric pressure of about 8.1 millibars, with a maximum of about 8.7 millibars and a minimum of about 7.5 millibars.

Maximum measured wind speed becomes important because it directly relates to available differential pressure. Vacuum chamber tests show that a persistent wind that dislodges small particles of grit must occur before any significant erosion effects can take place. This is because without small particles to strike and dislodge local soil grains (a process called "saltation") the wind has little ability to carry enough particles to erode the sediments.

Surface winds on Mars usually run from 16 to $32 \mathrm{~km} / \mathrm{hr}$ with measured peaks of $113 \mathrm{~km} / \mathrm{hr}$ by Viking. At an air pressure of 8.1 millibars even this could produce the erosion effects over time. This means that atmospheric effects could be the driving force. However, when we consider the difference in atmospheric pressure on Earth and the pressure at the Opportunity sites, we can see that there is literally 125 times the amount of force behind a breeze at any given velocity on Earth, based on the volume of air molecules moving past a given point.

A cubic meter of dry air on Earth at STP has a mass of 1.2754 kilograms, while a cubic meter of dry martian atmosphere at this Opportunity site would have a mass of 10.33 grams. This implies that overall, only a tiny fraction of the force would be available not only to work against a rock face but also to carry the grit needed to perform the abrasion.

Liquid water, while controversial, is know to be present for brief periods, often in the form of frost, fog, or dew. While water has a much greater capacity for driving these erosion effects, we would have to see evidence that it is present in sufficient quantity. Having much more mass in a concentrated area, and therefore, much greater kinetic energy potential, water-borne grit would be an effective means of creating the stems.

Given the density of liquid water versus that of the martian atmosphere, it is easy to calculate that a given volume of water spray would deliver 784 times as much force as an equivalent volume of moving martian atmosphere. From this perspective it is clearly much more effective as a force providing agent for driving erosion effects just on a pure mechanical basis alone.

Finally, it may be possible in some circumstances for liquid carbon dioxide to be present on Mars for brief periods of time. The question is whether it can form readily enough and for enough time to act as a carrier fluid for abrasive grit. The formation of liquid CO2 is not as simple as it sounds however. While the night time temperatures can reach low enough values to allow it to exist, the nearvacuum conditions would inhibit this under most circumstances.

It is known that dry ice snow has been observed to fall by the Phoenix lander ${ }^{\text {vii }}$, although it was not able to reach the ground as it sublimated into a vapor quickly. Based on this observation, it is not very likely that this fluid is responsible for grit-driven erosion episodes.

Other fluids are not credible due to the fact that any substance that could become a liquid or exist as a liquid on Mars would leave a distinct signature that would be detectable through spectrographic or other detection methods. We see no sign of this.

This only leaves wind and liquid water as our possible erosion agents.

\section{Physical evidence of stem formation processes}

The morphology and structure of the stems are an important part of the puzzle but we also can observe a number of other clues in the immediate environment of each group of stems. The first clue to take note of is the orientation of a group of stems. This image from Opportunity, sol 85, (Figure 2) shows a distinct group of stems that point in one direction. Our first assumption would be that this is affected by a stable, prevailing directed stream of erosive material. 
This is an accurate concept with one exception. We can see that the adjacent face of the boulder has another group of stems that are parallel, but they face an entirely different direction. And, in another image, a different face of the same boulder has a third distinct population of stems that are parallel but again, oriented in another, unique direction.

This raises an immediate question- what is this force that operates independently on each face of the boulder, and clearly must be capable of exerting itself for a duration that allows clear, deep erosion to produce the stems? We must discount wind as the possible agent at this point. After all, a single prevailing wind movement is a credible hypothesis, but three independent streams of wind coming at the boulder, depending on the face of the boulder, does not make sense.

Here we must look for something that will entrain or collimate the wind movement for days, weeks, or even years, and will also somehow aim itself in three consistent directions. Or, we could look for independent forces that achieve the same end without introducing complexities. There is another force that can do this. The topography of the immediate area will show an answer.

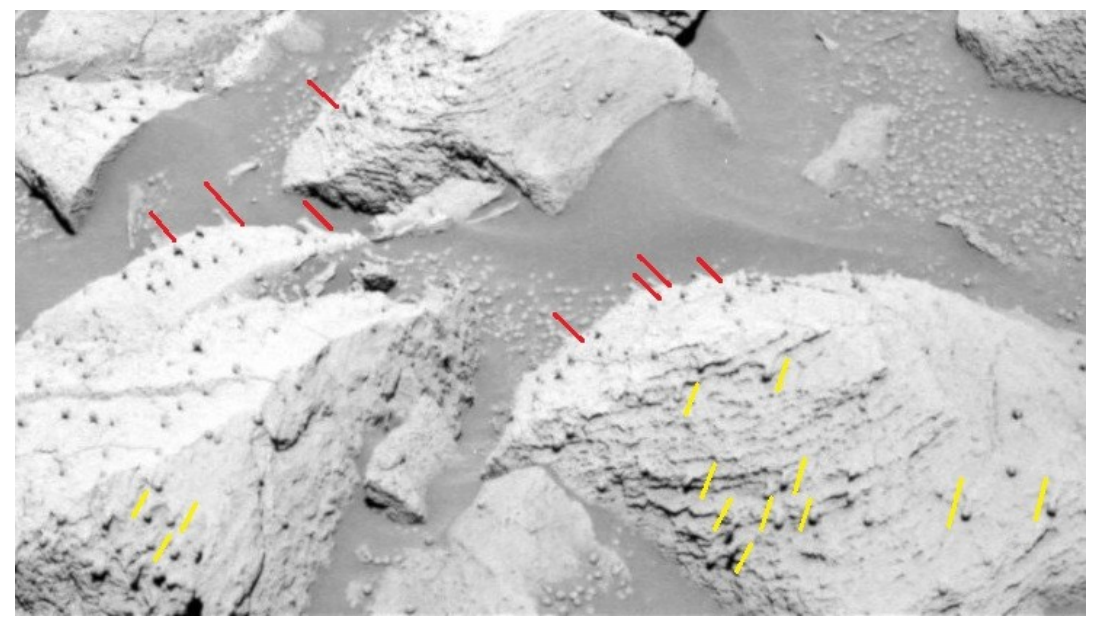

Figure 2 - the red lines show alignment vectors for one population of stems on similar faces of two rocks. Likewise, a second population of stems on both rocks also align to a common vector (yellow) from a completely different direction.

Wind is not this selective, and therefore we must look to another force that is creating this effect.

\section{Site layout and erosive force pathways}

If a site map is created from the rover data, it becomes clear that common features emerge in association with the stem structures. Image data from numerous imagers and over the course of many different sols have provided a map with numerous perspective points, allowing an examination of the potential flow paths of the force carrying fluid.

Using six image frames, this site map has been assembled that includes the features of interest. Each feature will be evaluated in order from end product (spherules on stems) to causa prime. The most extreme (and well-aligned) group of stems can be seen in sol 164 images inside the boundary of Endurance Crater. 


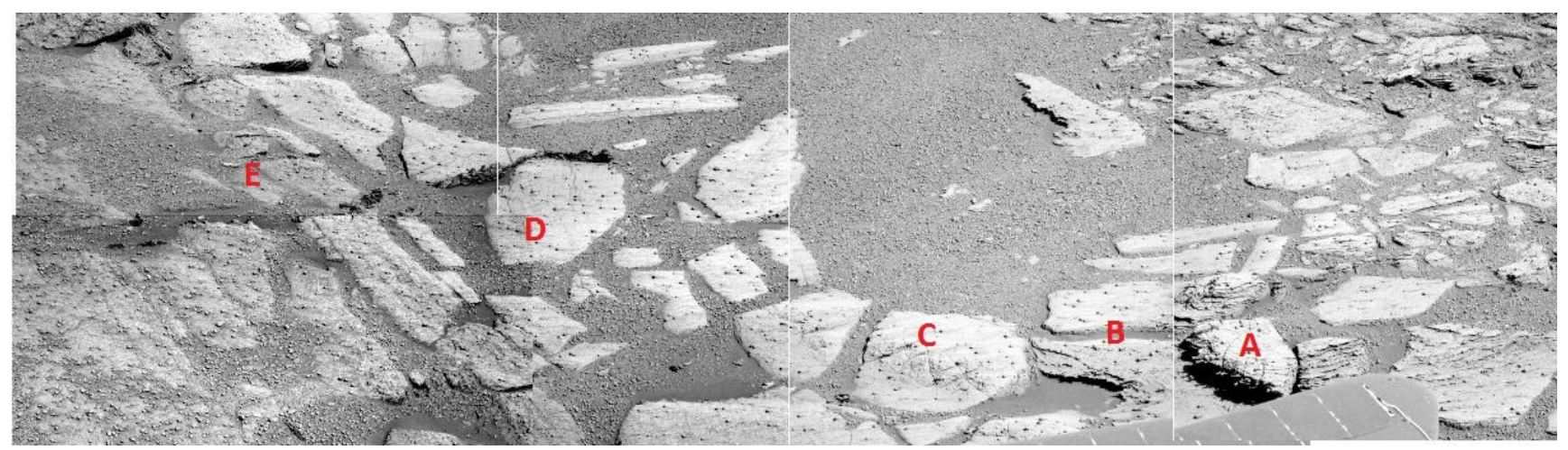

Figure 3 - the site map for this study. This map is a composite from Opportunity rover images ranging from sols 139 to 165 . The red letters identify each zone that will be addressed in this paper.

\section{Zone A - the stem grouping}

This is the target area of the erosion. Numerous stems are present on the larger rock mass, and all are oriented in the same direction (to the left of image). Stereo views show the elongation and structure clearly, and the areas of rock with no spherules also show a great deal of rounding and literally no sharp edges or surfaces.

The rock in question appears to be the target for a consistent and directed stream of abrasive. Clearly some fluid, whether air or water, has carried it and formed the stems. A view of the stems shows very clearly that the force was applied in a single direction. Examining the area directly behind the rock shows that a slab behind it also shows wear as if the working fluid has affected it as well. This is to the right of the stem rock image.

Examine Figure 4 and it will be clear that the layers of sediment consist of more durable material separated by some softer material. The erosion wears this softer material away, enhancing the edges and layers in the slabs. Notice also that other slabs which have not been exposed to this force show little variation with the layers being less visible. 


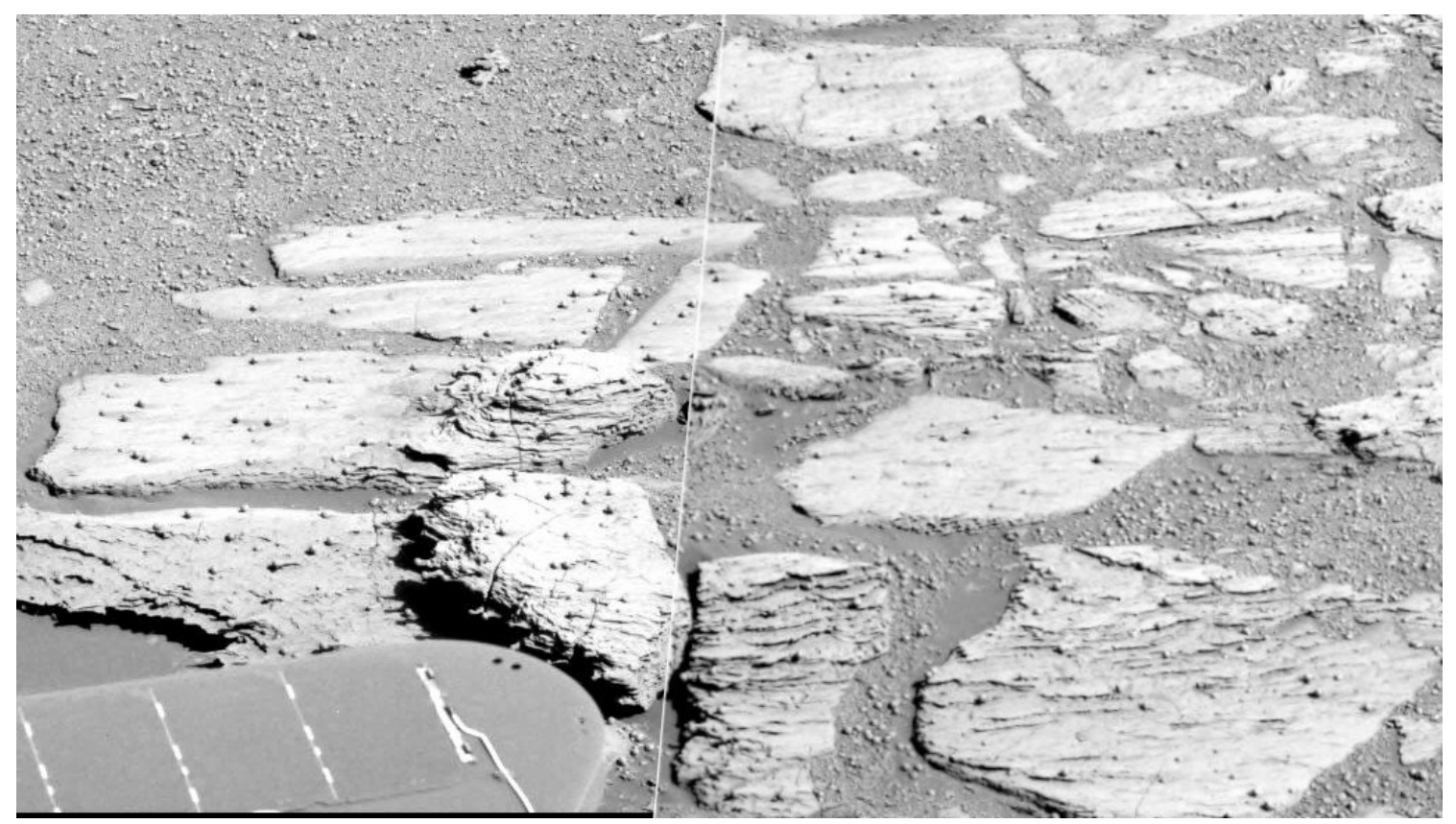

Figure 4 - Zone A shows the evidence of erosion with the "stem stone" above the rover solar panel, left of center of the image. All stems are pointing at the source of the erosion. Behind this stone, to the right, we see that the rock slab has also been affected, leaving the sedimentary layers worn away and very visible.

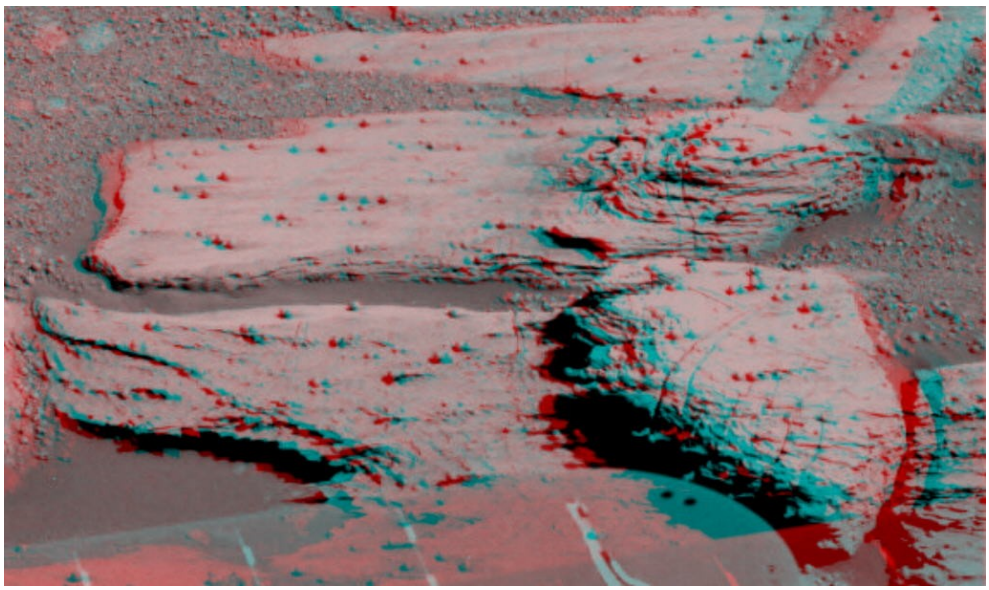

Figure 5 - a stereo anaglyph of the stem area showing their alignment. This image leaves little doubt that the erosion was directed in some manner. It also yields evidence that the sedimentary layers are separated by somewhat softer material than the body of each layer.

This image is from sol 164, just inside Endurance Crater.

\section{Zone B - eroded foundation slab}

In this zone it is clear that the stone slab has been worn so that a large chunk is missing from the left edge. The wear is an oval "bite" taken out of the slab, indicating that the force was greatest or more persistent at the center of this region.

This slab also shows aligned stems that match those of the target area. Similar erosion can be seen beside the large stone in Zone A, which shows both types of erosion- the flat stone has edges enhanced while the target stone at the end shows extreme layering and some small stems aligned into the supposed flow area. 
This can all be seen in Figure 5 in the anaglyph. Less of the force was applied there but the effects, while diminished, remain clear.

\section{Zone $\mathbf{C}$ - worn channel in stone}

In this image we can see a very clear demonstration that the erosive flow was persistent enough to literally cut a trough or channel into the stone slab. The path of the force is apparent from this image. See Figure 6 for an anaglyph showing the true geometry of the flow path.

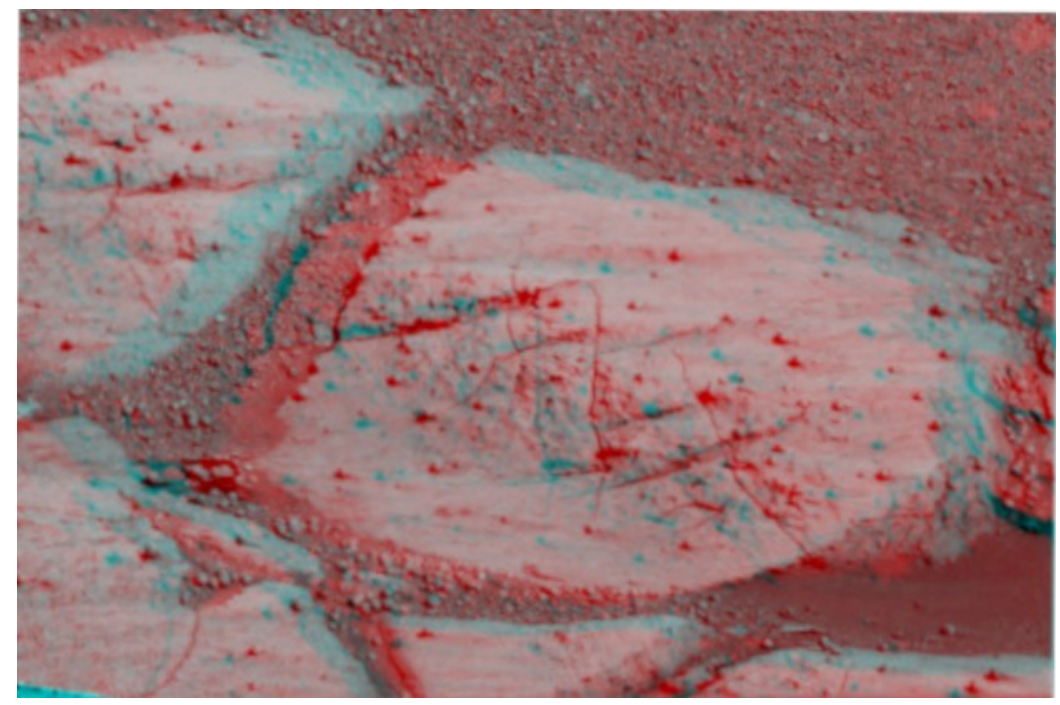

Figure 6 - an anaglyph showing that the erosive force has cut a channel through the stone slab in front of the target zone.

This channel traces the path of ejection backwards to a specific area. Along that path we can see signs of water erosion due to the features being something that wind cannot logically create.

\section{Zone D - deformed stone slab}

Moving back still further toward the source of the erosion, something unusual shows up. The stone in this area is actually deformed like clay. This is something that requires a great deal of tightly focused force, and also has implications for the stone material itself.

It has been theorized that this material is the sea bottom that has acquired layers from sedimentation, but also that when the sea vanished, this sea bottom mud dried and split into large stone polygons. This effect can be found in many locations on Earth, such as Death Valley.

In those cases we have a clear picture of their source and history. They were definitely formed from a dried sea bottom and we have direct physical samples that have been analyzed. The visual characteristics of both Death Valley and Meridiani Planum are identical in this respect: they are both fields of mud polygons that are the remains of mud from salty bodies of water that dried up in the past.

Furthermore, we know that when water is applied to the mud polygons in Death Valley (as well as other areas), a slick clay forms that can be molded and shaped with relative ease. But there is a caveat- the clay must remain wet for extended periods of time to show this character. The deformable nature of this material implies that it must have remained wet for weeks or months to be able to take on this signature shape.

When a high-pressure, persistent spray of water is applied to this material, it can deform into precisely the shapes that we are seeing in the sol 164 images from Mars. Under lesser pressures and less persistent water, this material will instead wear into troughs or channels, exactly what we see in Zone C. This is because it is harder due to the lower moisture content. Instead of deforming, it will erode. 
If we follow this reasoning we can see that the area near a persistent source of water would in fact be softer and more clay-like. Areas further from that source would be harder and would erode away instead of deforming. Areas that are much dried would act more like stone and would erode into stems if spherules or other hard objects shielded the surface from grit and water spray. See Figure 7 for an anaglyph and to follow the path of erosion.

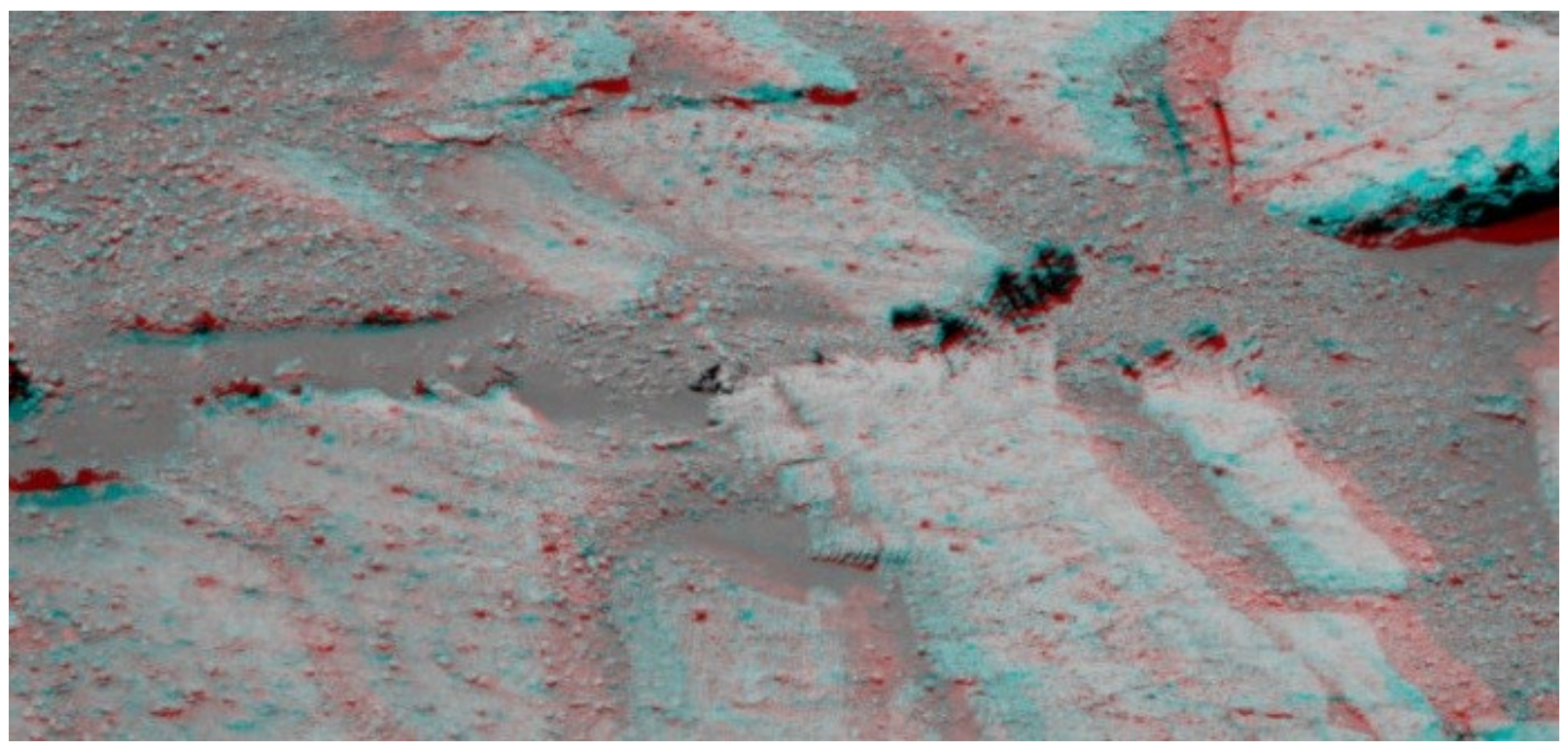

Figure 7 - clay fins created by wet clay being struck by a pressurized stream of liquid over time. This liquid would be capable of carrying grit and abrasive, thus shaping the features that we have seen, up to and including the stems themselves. Zone D is to the right, zone E to the left. Image from sol 162, Opportunity.

\section{Zone $\mathrm{E}$ - discharge zone with fins}

Now we have come at last to the area which appears to be the source of the erosive force. Wind, as stated previously, cannot perform these actions as it does not have the persistence of direction, focus of power, or ability to bend stone in one location while just bare meters away, it will literally scour it away.

The differences are in the properties of the stone itself, and of the dissipation of a focused and directed force. One and only one force meets the requirements, and that is of a liquid which can soften clay, carry abrasive material, remove debris, and work in a restricted stream or cone.

Furthermore, only one liquid that is present on Mars will meet these requirements, and that is liquid water. Given the simple visual evidence alone, we can deduce that there must be a functional water table, at least in this area of Meridiani Planum.

Figure 8 provides an image of the immediate area where the water source must exist. We see clear and proven evidence of water presence in how the mineral surfaces have been modified. Fins of minerals appear and there is even the visual evidence of dried salts where pools of brine would have evaporated or drained away. See Figure 8. 


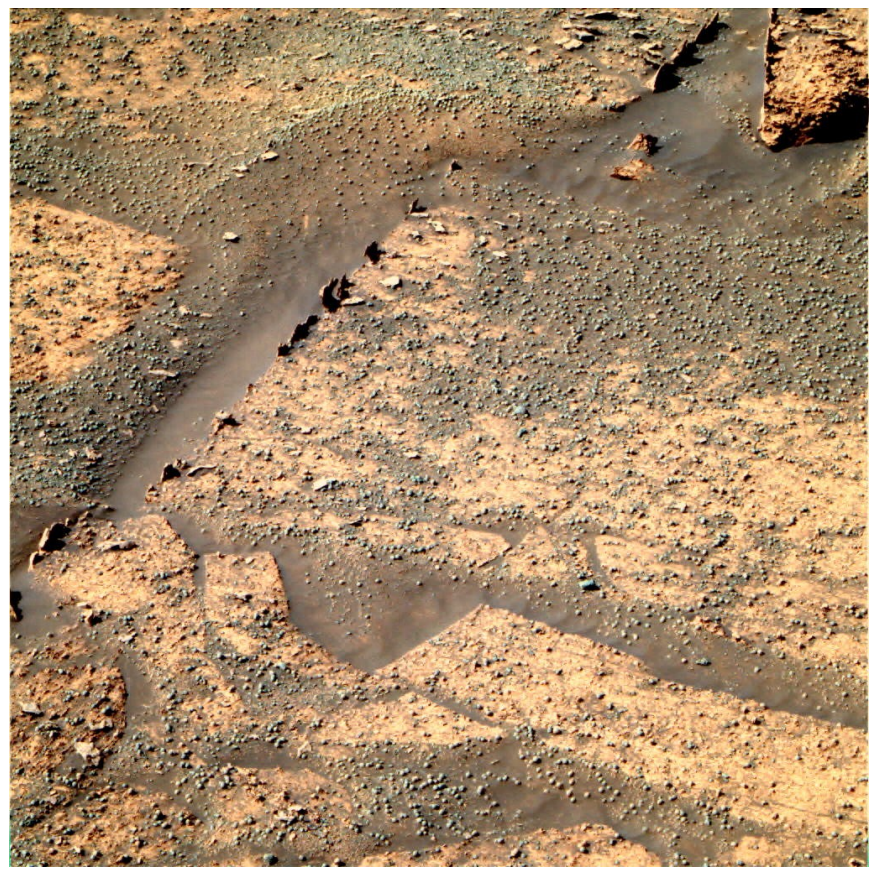

Figure 8 - evidence of water presence is shown with the mineral fins and the areas that appear washed clean of spherules and debris. This area is known to contain a large quantity of sulfate salts from the dried ocean that was present in the past.

Now we can see where the "high water" mark was and how it exhibits what appears to be a line of dried salts (upper left of image).

The formation of these features cannot be performed by wind. It takes a long-term presence of liquid water to dissolve and then deposit this material.

Image from sol 162.

\section{Implications for water spray}

If we assume that water spray is being produced by geysers or openings in the water table, we can see that droplet spray on Mars will act very differently than droplet spray under terrestrial conditions, i.e. STP. This is due to the drag coefficient for dense air versus near-vacuum. In air at STP a spray of droplets will begin to lose energy and dissipate almost immediately on ejection.

Droplet size itself is the culprit because a droplet half the size of a given droplet will have only $12.5 \%$ of its mass but $25 \%$ of its surface area. All things being equal, the smaller droplet will decelerate twice as rapidly and therefore be rendered ineffective very quickly. Droplets of small size transfer their momentum to the surrounding air and create turbulence and eddies in the flow. But if we are in extremely thin air, drag forces become negligible and now the droplets will reach their target with nearly undiminished force.

This implies that if cold, pressurized water spray, loaded with sand particles, is ejected toward a rock face, the particles will be delivered with much greater force than they would be on Earth. Add to this the fact that in 38\% of the gravity of Earth, water spray on Mars will travel farther before falling to the soil. The effectiveness of geyser spray on Mars would be far greater than an equivalent event on Earth.

\section{How often would these events occur?}

One positive factor is that the rover often images the same area for numerous consecutive sols. If we take two images of the same area, we can subtract the image data from a static frame and find the differences. For example, the same area was imaged on sol 139, sol 152, and sol 162 . While the location of the MER was different (or more precisely, the location of the PanCam was different), we can (for example) convert the image data for sol 152 into a negative and add it to the frame for sol 162.

If anything has changed in the frame, such as the movement of spherules or sand (or even shadows), we will be able to see that immediately. Examining the two sols, it is clear that no eruption has occurred within that 24 sol period. Virtually every feature remains unchanged. It is possible that 
geyser output, if it is occurring, happens on a seasonal basis or is only sporadic. Changes in season also bring temperature and air pressure changes as well. This could influence the activity of geysers in a low-pressure environment.

\section{Can liquid water even exist on Mars?}

The short answer is “yes”. What nearly every researcher misses is that the models are based on the tables showing the properties of distilled water. In the real world this is almost never present. Water on Mars would have many contaminants including an extensive menu of salts, mostly sulfates, and fine dust and powder from the environment. Salty water can often survive extremes of temperature, and because of this, brine is often used in industrial cold storage systems as the heat transport fluid.

This also changes the boiling point and ease of evaporation. Experiments performed in laboratory settings prove that the rate of evaporation on Mars can be very comparable to what we see on Earth, with rates of 0.8 to 1.1 millimeters per hour being common ${ }^{\text {viii }}$. Controlling factors are not just temperature and pressure, but also advection, where local air can become so loaded with moisture that further evaporation cannot easily occur.

In a sense, the water vapor becomes a part of the local atmosphere and prohibits further loading. This fact is supported by actual measurements showing that the atmosphere on Mars contains ten times more water vapor than theory can predict. Given these facts, it now seems much more reasonable to assume that the soil and rock can be wet for extended periods of time, and that geyser action becomes far more credible as a result.

\section{Is liquid water present on Mars today?}

To answer this question, let us look at data from two distinct sources.

First, we know that erosion occurs on Mars on a day-to-day basis. Images of sand that has impressions of the Mossbäuer spectrometer plate show that overnight, the sand has completely moved, nearly erasing the print. Further images from sols 199 to 123 show that some impressions of the spectrometer plate were actually made in extremely cohesive soil that stuck to the plate when it was removed.

The soil in the area showed a very distinct change in its location and arrangement over a single night, and when impressed, it bulged like mud, not at all like sand. The rate of erosion and the consecutive images from the rover prove that the features we are now observing had to be formed within the last year or less. These features are changing and being remade on a constant basis. Image data has documented and proven significant changes happening overnight, and in some cases those changes would require an active liquid to take place. See Figure 9.

\section{Final notes}

This research has located and identified approximately 40 geysers or water drainage holes in the Meridiani area alone. They are characterized by "blown back" material, water-worn slots, cones of ejected soil and spherules, and other features such as strong infrared absorption at 1013 nanometers in the imager data (filter R7).

This image from sol 146 (Figure 10) is perhaps one of the most convincing. We can clearly see what appear to be water trails on the sand. This image has been made in stereo and the right pancam filters show an increasing absorption of infrared with increasing wavelength, which is consistent with the properties of liquid water. 
In conclusion, the most logical answer is that the spherules on stems were formed by the action of liquid water geysers ejecting grit-laden spray at the rock faces. This process is ongoing and clear evidence shows that it is very likely happening now.

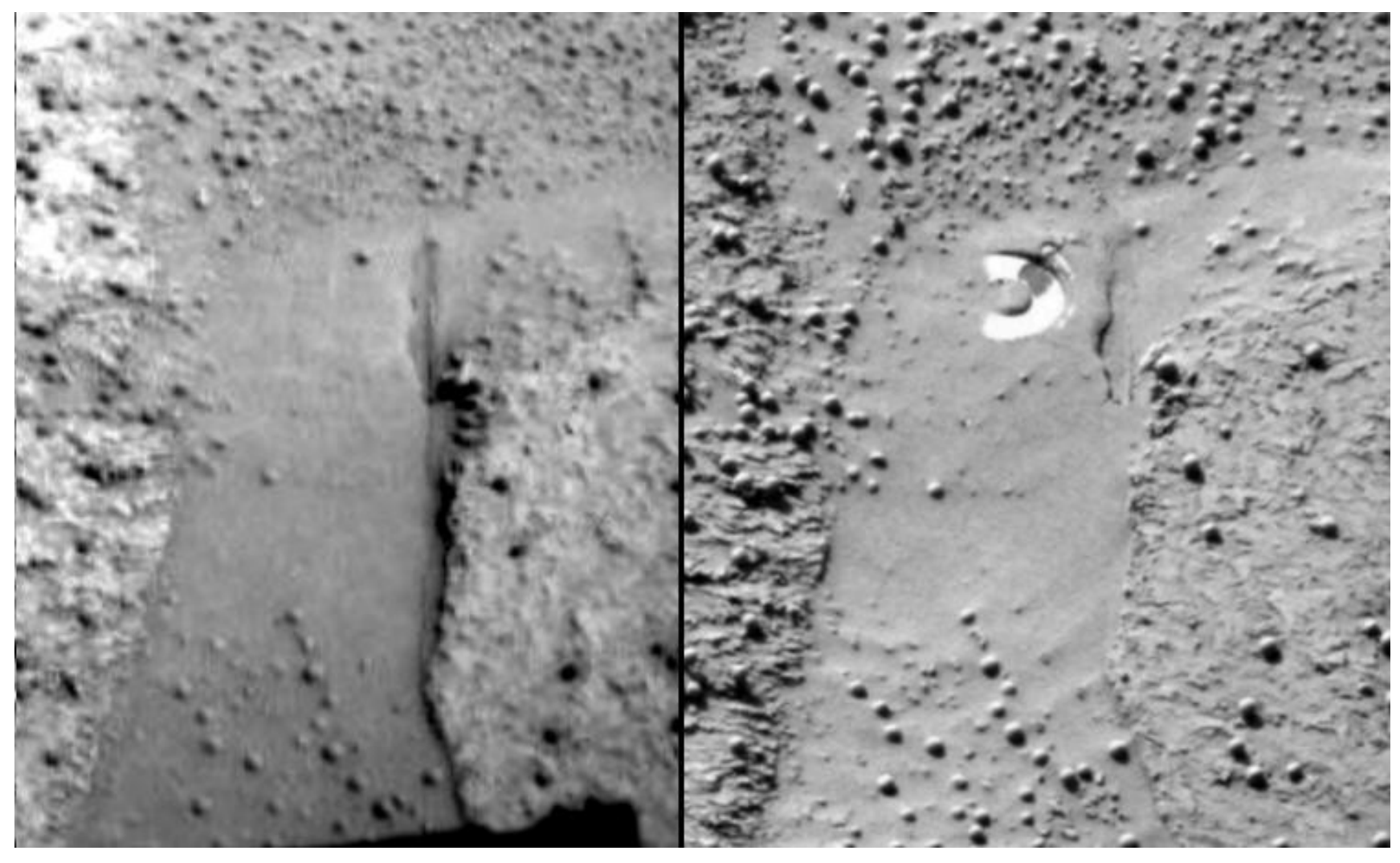

Figure 9 - active changes requiring liquid water overnight. The left image is from sol 119 and shows a thin channel in the soil aligned with the left edge of the right-hand stone slab. The right image shows the circular impression of the Mossbäuer spectrometer plate, which made a bulge in the soil. This implies that it is mud, not sand. And, the channel along the right-hand slab has now changed, ending very near the circular impression and no longer following the contour of the slab. 


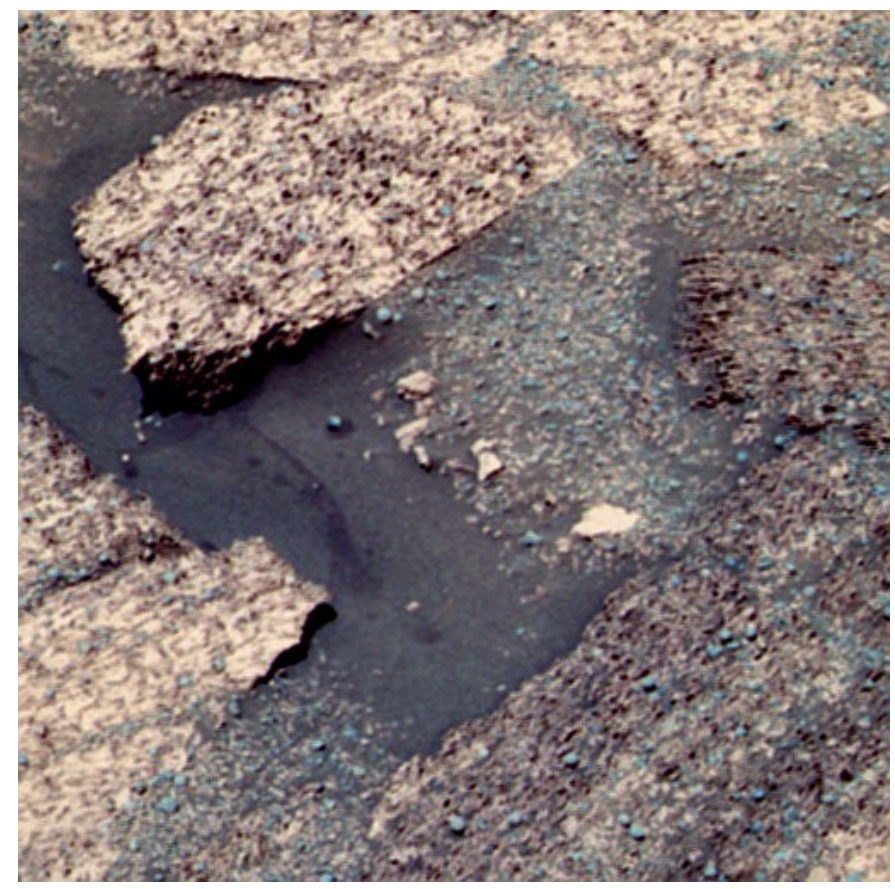

Figure 10 - what appears to be liquid water trails on the soil in Endurance Crater. Note the liquid is under and around the spherule and small rocks on the sand.

In stereo the features are persistent and appear to be from a slowly decreasing flow of liquid. Spectral data shows an increasingly dark set of markings as the wavelength increases. This is consistent with the absorption of infrared by liquid water.

Image from Opportunity, sol 146.

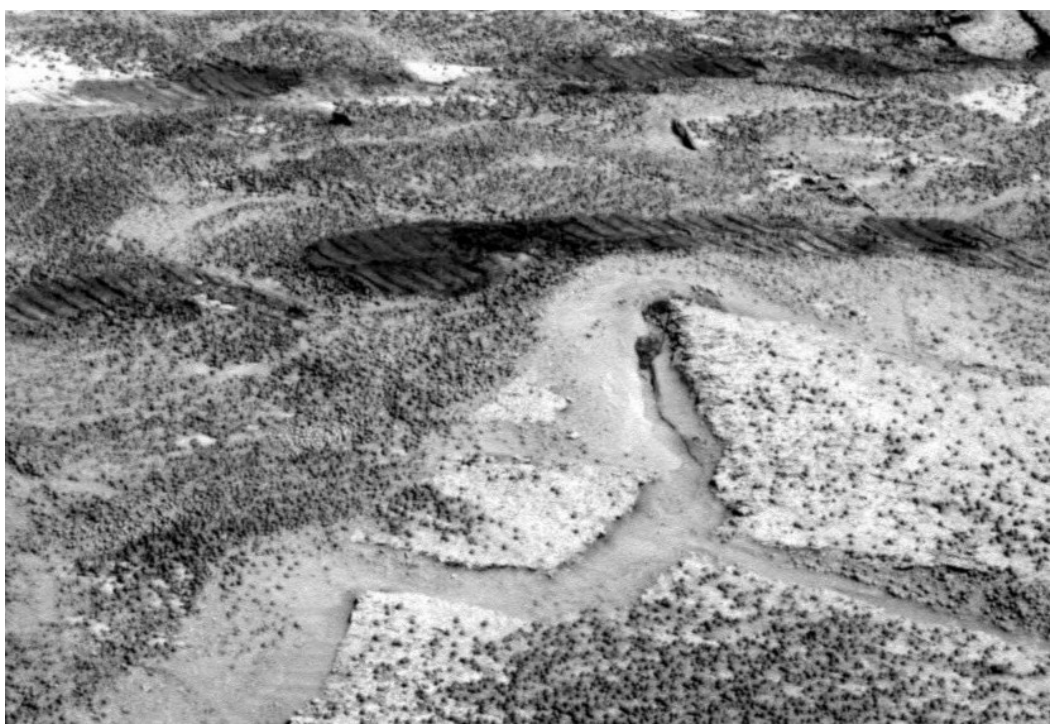

Figure 11 - an apparent geyser from sol 122. The rover tracks are dark as if muddy, and the geyser has piled up sand and detritus in this image. NASA states that the piled up cone of soil and spherules at the top of the feature is due to "soil subsidence". This statement is clearly incorrect as soil is not known to subside uphill. Anaglyph images show the ejecta cone as being piled higher than the surrounding soil. Note the clean runoff areas where no spherules exist. 
i https://www.researchgate.net/publication/

351252619 Fungi on Mars Evidence of Growth and Behavior From Sequential Images

ii Hematite spherules at Meridiani: Results from MI, Mini-TES, and Pancam,

W. M. Calvin, J. D. Shoffner, J. R. Johnson, A. H. Knoll, J. M. Pocock, S. W. Squyres, C. M. Weitz, R. E. Arvidson, J. F. Bell III, P. R. Christensen, P. A. de Souza Jr., W. H. Farrand, T. D. Glotch, et al

Journal of Geophysical Research, Planets, first published: 04 December 2008 https://doi.org/10.1029/2007JE003048

iii Fe-oxide concretions formed by interacting carbonate and acidic waters on Earth and Mars, H. Yoshida1, ${ }^{*}$, , H. Hasegawa1,2, ${ }^{*}, \dagger$, N. KatsutaI. Maruyama, S. Sirono, M. Minami, Y. Asahara4, S. Nishimoto, Yamaguchi, N. Ichinnorov and R. Metcalfe; Science Advances research article, Vol. 4, no. 12, eaau0872, 05-December 2018

iv Giant Polygons and Mounds in the Lowlands of Mars: Signatures of an Ancient Ocean? Dorothy Z. Oehler and Carlton C. Allen; AstrobiologyVol. 12, No. 6, Hypothesis Article Published Online: 13 Jul 2012 https://doi.org/10.1089/ast.2011.0803

v CHARACTERISTICS OF BASALTIC SEDIMENTS REVEAL DEPOSITIONAL AND TRANSPORT PROCESSES. Robert A. Craddock, Tim Rose, Kathleen M. Marsaglia, Jon Cawley, and Alex Morgan 50th Lunar and Planetary Science Conference 2019 (LPI Contrib. No. 2132)

vi The Metric Atmospheric Model of Mars, https://www.grc.nasa.gov/www/k-12/airplane/atmosmrm.html

vii NASA observations point to dry ice snowfall on Mars, https://www.jpl.nasa.gov/news/nasa-observationspoint-to-dry-ice-snowfall-on-mars

viii On laboratory simulation and the temperature dependence of the evaporation rate of brine on Mars Derek W. G. Sears, Julie D. Chittenden; Geophysical Research Letters, Volume32, Issue23, December 2005 First published: 14 December 2005 https://doi.org/10.1029/2005GL024154 